\title{
LEAP MOTION CONTROLLER: A VIEW ON INTERACTION MODALITY
}

\author{
Anish A Aziz ${ }^{1}$, Reshma K ${ }^{2}$ \\ ${ }^{1}$ College of Engineering Vadakara Kozhikode, Kerala, India \\ ${ }^{2}$ College of Engineering Vadakara Kozhikode, Kerala, India
}

\begin{abstract}
Changes are the basis for innovations. The types of user interfaces to devices are changing day by day. The users can choose the best input modality. Kinesics based user interfaces, combined with the current techniques impart naturalistic current chance for unambiguous operation, for people who are not comfortable with predominantly used input devices and technology. That is extending the existing system of touch pad, touch screen etc. by the idea of controlling a PC or laptop by sensing human gestures is of great relevance. This is what we were intending to do with the Gesture based PC control using Leap Motion controller. For this purpose we plan to extend the usage of Leap Motion Controller by assigning different gestures as input for performing various operations as we prefer. The device receives hand motion and finger motion as input which uses infrared (IR) ray to determine the position of objects in a delimited space.
\end{abstract}

Keywords: Gesture Based User Interfaces, Leap motion controller, IR Imaging, API. $* * *$

\section{INTRODUCTION}

The interaction modalities of user interface play adominant role in the relation between people and computer technology. The user's approach of using interfaces has undergone a radical change. Majority of the population is using touch devices. Gesture based user interfaces like Leap Motion Controllers, Kinect sensors etc are also now available in the market. There is a possibility for extending the existing system of touch pad, touch screen etc completely by such devices. Here we extend the PC Control using Leap Motion controller. We use Leap [1]device since the studies showed Leap Motion Controller is more accurate than the similar other motion sensor [2] devices. Leap Motion Controller is mostly used for gaming purposes. We try to extend its operations so as to use it instead of mouse events. We use a Leap Motion Controller which is a most modern device currently used for gaming, trying to use instead of keyboards by implementing on screen keyboard. Various gesture tracking devices are available viz. Eye tracker, Vision-based hand tracking system, wearable motion sensors, Mobile text input devices etc.. These are widely used for implementing virtual reality or augmented reality[7].

\section{RELATED WORK}

As specified it is planned to develop a technology such that we can control our PC without using a mouse or hand touch but merely with hand gestures. The different objectives that tried to implement are as follows. To do the operations of mouse such as right click, option selection and pointing operations with hand gesture. Applying screen lock by using drawing patterns, sliding operations for the easy usage application software and shutdown operation, copy-paste operations by using drag and drop methodology etc. For the above operations zooming or cropping off images, on-screen key board for writing are also to be implemented by hand gesture recognition. For more accurate operating we liked to use a pen like sharp edged tool instead of hands. We find it is very challenging to set programs using the deviceleap motion controller, since it is completely a new technology. Here we tried to replace the mouse events. Mouse pointer movement and mouse click were implemented.

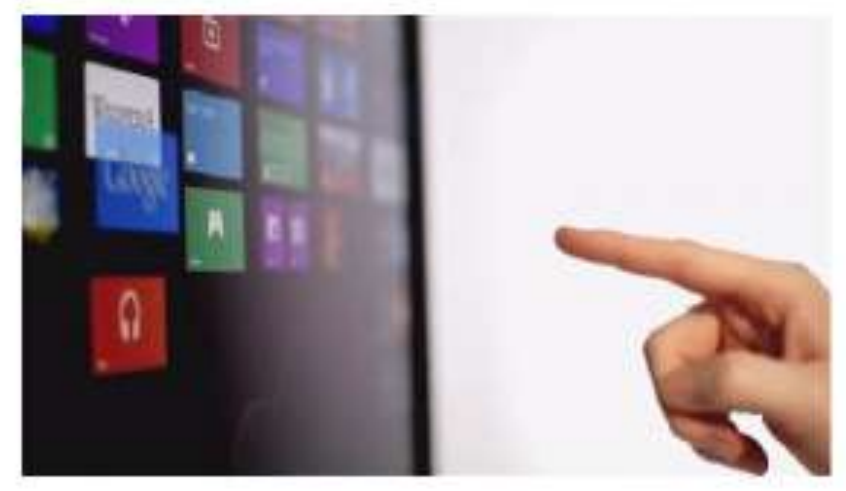

Fig 1: Generic view of leap gesture

The Leap Motion Controller is placed roughly $20 \mathrm{~cm}$ in front of the PC, so that it covers the range of hand movements.

\subsection{Leap Motion Sensor}

Leap Motion Sensor is yet another recent sensor which is based on vision techniques and employed to capture the hand signs in $3 \mathrm{D}$ digital data. It is a small device with 
sensors which report data such as position and speed of palm and fingers based on the sensor's coordinate system. Data are transmitted to a computer via a USB connection. The sensor is placed next to the keyboard on our work desk .It provides very accurate individual finger detection and differs from the Kinect by providing a 3D description of the framed scene. Compared with Kinect sensors, it produces a far more limited amount of information and works on a smaller 3D region, but the advantage is that the captured data are precise and accurate (accuracy of about $200 \mu \mathrm{m}$ ). The main intention of Leap Motion sensor is precise finger detection and thereby integrating these functionalities with existing applications.

\subsection{Kinect Sensor}

The Kinect Sensor [4] is what we call a "depth camera" it is a combination of a normal digital camera and second sensor that reads the distance to each pixel. It functions by covering the room with a constant, predetermined pattern of infrared dots. The monochrome CMOS sensor is placed at an offset relative to the IR transmitter, and the difference between the observed and expected IR dot positions is used to calculate the depth at each pixel of the RGB camera. Kinect sensor uses a unique method to captures depth image. The capturing is done by using two separate data streams by which the first stream presents the data from the Kinect sensor to the nearest object in millimetres whereas the second one present the segmented data from a tracked person.

\section{FEATURES OFLEAP MOTION}

\section{CONTROLLER}

Leap Motion Controller is a USB device that works based on infrared technology by receiving hand and finger motion as input. It is similar to a computer mouse and uses infrared imaging to analyse the position of objects in a restricted space to a distance of about 1 meter. Here three different Infrared LED emitters are used along with two Infrared cameras as shown in the fig 2.The IR diodes generates spots of Infrared light which forms a pattern and using the rectified data cameras generate almost 300 frames per second, which is then sent through a cable to the connected computer. A gesture listener isto be designed to support the Leap Motion Controller, to control and interact with a Java application and thereby to implement this work. The leap sends frames to the computer with maximum speed, where the number of fps can vary between specified ranges. Every frame can have several numbers of objects; every object type can have separate collection of object. Every object can be described by collection of parameters: usually positions in space. For this, the Leap Motion API uses a 3dimensional coordinate system, with $\mathrm{X}, \mathrm{Y}$ and $\mathrm{Z}$ axes as shown in figure 3 given.

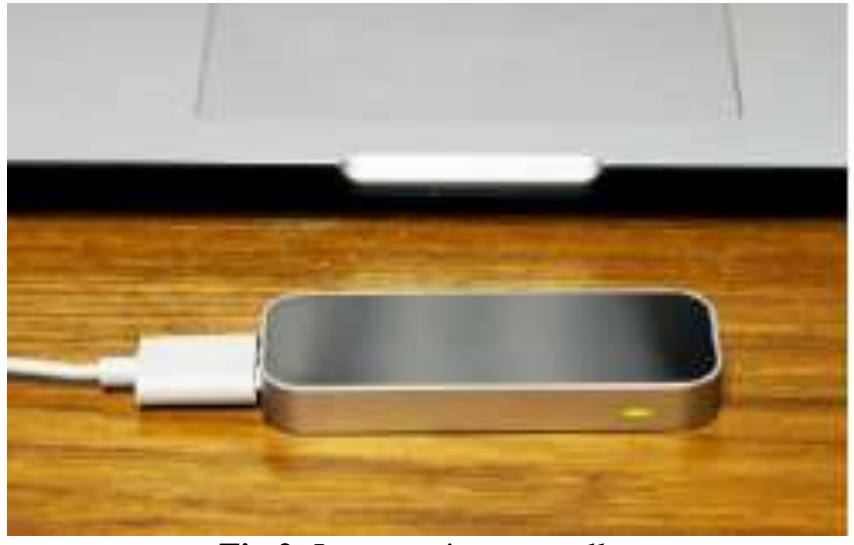

Fig 2: Leap motion controller

Where the centre is at the middle of the device, the Y-axis is perpendicular to its surface (vertical, positive values up), and the $\mathrm{X}$ axis is along the long axis, while the $\mathrm{Z}$ axis is perpendicular to both, positive values increasing away from the screen (if the device is parallel to the screen). The Leap uses the centimetre as the unit of measurement.

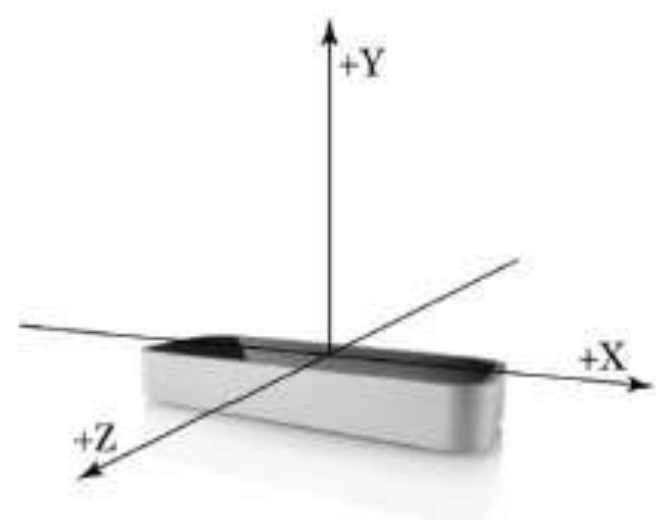

Fig 3: Leap Motion Controller Coordinate Axes

\section{INTERNAL WORKING}

Connecting the Leap Motion Controller and the PC requires SDK and Leap Motion Software downloaded from leap motion developer site. As mentioned earlier, our goal is to control a PC using different hand gestures. We could control the system viz. Copy-paste, drag and drop, shut down the system, opening and closing files/folders etc... In order to implement this we did the Calibration[10] process. It is in Calibration process, we set the coordinates of the screen. i.e here we set the coordinates of the window with that of the Leap Motion Controller Device. It is possible to use the hand gestures only after setting up the coordinates. Once when the coordinates are ready we, we can map the sensed hand gestures to the window. We set the coordinate value $(0,0)$ as the top left portion of the window. 


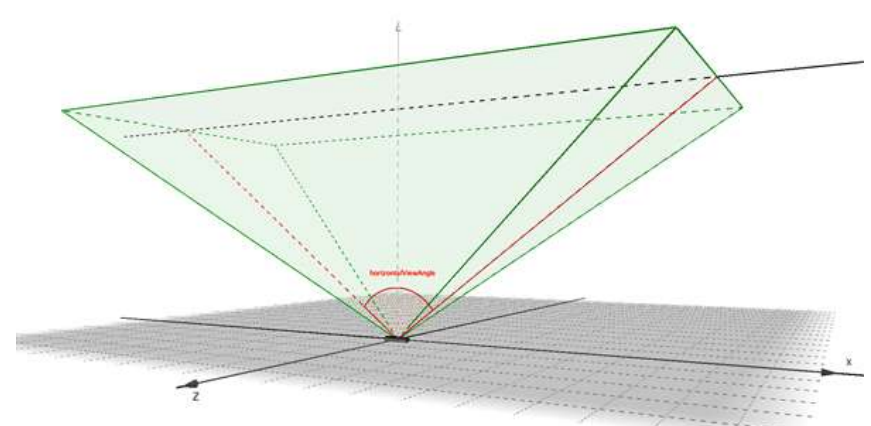

Fig 4: Range of Leap Motion Controller Device

Different hand gesture movements[12] are sensed by the Leap Motion Controller, if the hand gestures fall within the range of the device as shown in Fig. 4.With the software we have developed as written, has support for mouse pointer movement by sensing hand movement in the space above the leap. Mouse click was also possible with same gesture sensing technology by evaluating the $\mathrm{z}$ - co-ordinate value change. For this we had to use robot package in java.

\section{FUTURE WORKS}

The device leap motion controller, with its enhanced interaction possibilities, provides an "absolute" position (at least five or six decimal places of accuracy, relative to the absolute position of the sensor itself). As this leap motion controller is the major part of our project which is having more possibilities of extensions and researches, our project can also have more and more future works. Instead of merely using it as an input device of gesture inputs for various operations we can think about connecting it with other devices such as VR mount, Data gloves or any other devices. We can combine the device Raspberry Pi and can extend the work for remotely controlling certain devices. Another good option may be to combine the speech recognition with gesture recognition for a more advanced technical environment. Leap Motion devices can be used in medical, industrial areas, where direct contact is required for controlling devices, when the person to control is engaged with some other activity. Developing a leap motion controller with more advancement, even possible to use it as a remote that is without cable connection to the system or even to control the system from far more distance which is currently not possible. People who are disabled can use this technology based applications[13].

\section{CONCLUSION}

Someday the youth may wonder that their old generation had used a hardware part called keyboard with their computers for several years. This device with its new unity assets can create a big makeover in the virtual reality environment. What if you could see through walls like superman beyond a tiny window of our senses, there is a vast world that we can't normally access, but that's going to change. At SVVR conference, leap motion CTO Davidholz talked about the fact that how the 3rdgeneration of Virtual Reality/Augmented Reality devices will pipe everything from ultrasonic depth sensing to IR night vision directly into our consciousness. Virtual reality may feel as flawless and mystic, however with leap motion control it feels spontaneous. Stringent certainty and improved flexibility are just beginning .The ultimate virtual reality with leap motion controller brings our vision to life with awesome demos. This high accuracy device can be used beyond limitations. We are sure that in the near future human will find some another method of interacting with computers and may start using newer interface approaches. People may extend the use of wearable devices for communications and other entertainments. Thus we look forward for a population using these gestural devices as common, natural, daily-use devices for day-to-day purpose in the near future.

\section{REFERENCES}

[1]. Leap Motion.[Online]. Available: http://www.leapmotion.com/

[2]. Joe Guna, Grega Jakus, Matev Poganik, Sao Tomai and Jaka Sodnik.," An Analysis of the Precision and Reliability of the Leap Motion Sensor and Its Suitability for Static and Dynamic Tracking".

[3.] Eduardo S. Silva, Jader Anderson O.de Abreu, Janiel Henrique P. de Almeida, Veronica Teichrieb1, Geber L. Ramalho1. "A Prelimi nary Evaluation of the Leap MotionSensor as Controller of New Digital Musical Instruments"

[4]. Tomoya Murata and Jungpil Shin The University of Aizu, Fukushima, Japan."Research Article-Hand Gesture and Character Recognition Based on Kinect Sensor".

[5]. Toshiyuki Kirishima, Member, IEEE, Kosuke Sato "Real-Time Gesture Recognition

by Learn ing and Selective Control of Visual Interest Points".

[6]. Juan P. Wachs, Student Member, IEEE, Helman Stern, Member, IEEE, and Yael Edan, Member, IEEE."Cluster Labeling and Parameter Estimation for the Automated Setup of a Hand-Gesture Recognition System".

[7]. Preliminary user experience framework for designing mobile augmented reality technologies Shafaq Irshad; DayangRohayaAwangRambli2015 4th International Conference on Interactive Digital Media (ICIDM) Year: 2015

[8]. C.Amma D.Gehrig, and T.Schultz. "Airwriting recognition using wearable motion sensors," in Proc. Ist Augmented Human Int. Conf. 2010.pp. 10:1 - 10:8 
[9]. C.Amma M.Georgi, and T.Schultz. .”Air writing: Hands-free mobile text input by spotting and continuous recognition of 3d-space handwriting with inertial sensors," in Proc. $16^{\text {th }}$ Int. Symp. Wearable Comp., 2012, pp. 52-59

[10]. Leap Motion, Documentation developers Guide - API overview, 2014http://developer.leapmotion.com

[11]. Gestural Technology: Moving Interfaces in a New Direction [Technology News]Lee GarberComputerYear: 2013, Volume: 46, Issue: 10

[12]. High-precision telerobot with human-centered variable perspective and scalable gestural interface Karl Kruusamäe; Mitch Pryor2016 9th International Conference on Human System Interactions (HSI)Year: 2016

[13]. First person movement control with palm normal and hand gesture interaction in virtual reality Chaowanan Khundam Computer Science and Software Engineering (JCSSE), 2015 12th International Joint Conference

[14]. Technology News, Gesture Technology: Moving Interfaces in a New Direction, Lee Garber 